\title{
Effect of foamability index of short chain alkyl amines on flotation of quartz
}

\author{
Sabina Szczerkowska ${ }^{1}$, Marta Siedlarz ${ }^{1}$, Marta Wojcik ${ }^{1}$, and Przemyslaw B. Kowalczuk ${ }^{1, a}$ \\ ${ }^{1}$ Wroclaw University of Science and Technology, Faculty of Geoengineering, Mining and Geology, Wybrzeze \\ Wyspianskiego 27, 50-370 Wroclaw, Poland
}

\begin{abstract}
Amines can be used for flotation of various minerals, especially quartz. The flotation efficiency of quartz depends on the amine type and dose. It was proved that the shorter alkyl amine, higher amine concentration has to be used to recover quartz at the same level. In flotation amines play a role of both collectors and frothers. The ability of a amine to collect particles can be expressed in the form of contact angle, while the foaming properties by different parameters including dynamic foamability index (DFI) and critical coalescence concentration (CCC). Determination of DFI and CCC requires advanced techniques and methods. Therefore, in this paper a rapid and facile method for determination of foaming properties of amines and also other surfactants was used. It was based on measuring the initial foam and froth heights in a conventional flotation machine at different concentrations of surfactants. The foam height-concentration curve was described by utilizing an empirical equation which was based on one-adjustable parameter called the foamability index $(F I)$. In this work the foamability index was determined for butylamine $\left(\mathrm{ButNH}_{2}\right)$, hexylamine $\left(\mathrm{HexNH}_{2}\right)$ and octylamine $\left(\mathrm{OctNH}_{2}\right)$ as examples of short chain alkyl amines. The determined foamability indices were 92,12 and $4 \mathrm{mg} / \mathrm{dm}^{3}$ for $\mathrm{ButNH}_{2}, \mathrm{HexNH}_{2}$ and $\mathrm{OctNH}_{2}$, respectively. It was shown that when the flotation results of quartz were presented in the form of recovery versus normalized amine concentration in relation to the foamability index $(c / F I)$, all the experimental data points converged to one curve. It indicates that amines act similarly but at different concentrations expressed as FI. The foamability index seems to be a useful parameter for characterizing any flotation frother.
\end{abstract}

\section{Acknowledgement}

This work was financed by the Polish Statutory Research Grant B50199.

\footnotetext{
${ }^{a}$ Corresponding author: przemyslaw.kowalczuk@pwr.edu.pl
} 